\title{
Off-pump connection of the pulmonary veins with bipolar radiofrequency: Toward a complete epicardial ablation
}

\author{
Stefano Benussi, MD, PhD, and Ottavio Alfieri, MD, Milan, Italy
}

1

lthough bipolar radiofrequency is presently the most reliable means to create transmural atrial scars, ${ }^{1-3}$ the clamping design of bipolar devices has limited its epicardial use to pulmonary vein (PV) isolation.

We describe a technique to perform a posterior left atrial connection (PLAC) between the 2 PV-encircling ablations epicardially, off-pump with a standard bipolar radiofrequency device.

\section{Technique}

The ablation technique is here described in a series of patients undergoing concomitant off-pump coronary artery bypass (OPCAB) surgery.

After median sternotomy and harvesting of the conduits, the pericardium is incised and suspended as for standard OPCAB. Heparin is administered. All grafts are completed during suction stabilization. Apical suction is used to enhance exposure. The left internal thoracic artery graft is tailored long enough to allow rightward displacement of the heart.

The ablations are performed with a bipolar radiofrequency device (Cobra Bipolar; Estech Inc, San Ramon, Calif), with the 64-mm-long ablating inserts mounted on a straight clamp. Radiofrequency energy is delivered for 40 seconds at $90^{\circ} \mathrm{C}$. All ablations are doubled to ensure electrical block. ${ }^{2}$

Dissection of the reflections is begun around the right PVs after developing the Waterston groove. To prepare the route of the PLAC, further dissection is carried out around the superior vena cava (SVC) to mobilize it from the right pulmonary artery and from the roof of the left atrium (LA). The right PV-encircling ablation is performed. Care is taken in keeping such encircling ablations on the antral LA and not on the PVs. The left pericardial traction sutures are pulled, and the heart is displaced rightward with apical suction. The reflections around the left PVs and the ligament of Marshall are dissected, and the encircling ablations are carried out. Maintaining the same heart exposure, the ablating device is then inserted with one jaw above and the other beneath the left PV couple. The clamp is kept widely open and slid toward the surgeon, with the upper jaw, the visible one, following the transverse sinus of the pericardium rightward beneath the ascend-

From the Division of Cardiac Surgery, S Raffaele University Hospital, Milan, Italy.

Received for publication Jan 12, 2006; revisions received Feb 17, 2006; accepted for publication March 7, 2006.

Address for reprints: Stefano Benussi, MD, PhD, Division of Cardiac Surgery, S Raffaele University Hospital, via Olgettina 60, 20132 Milan, Italy (E-mail: stefano.benussi@hsr.it).

J Thorac Cardiovasc Surg 2006;132:177-8

$0022-5223 / \$ 32.00$

Copyright $\odot 2006$ by The American Association for Thoracic Surgery

doi:10.1016/j.jtcvs.2006.03.012 ing aorta and the SVC and the posterior jaw moving freely around the posterior-inferior left atrial wall. Care is taken not to force the clamp against any unseen resistance. The apex of the heart is let down, and the SVC is gently retracted toward the left to expose the tip of the upper jaw of the clamp. The table is tilted in a Trendelenburg position to optimize venous return. The clamp is closed, making sure that the upper jaw ends up biting the right encircling ablation with its tip (Figure 1). Also, the proximal end of the jaw must be checked for contact with the left encircling ablation. Radiofrequency energy delivery is started. Thanks to the incomplete obstruction of the pulmonary venous drainage (Figure 2, A), the maneuver is well tolerated, with its hemodynamic effect generally not unlike that caused by clamping around a PV couple. The resulting set of lines is sketched in Figure 2, $B$. The blind positioning of the posterior jaw leaves room for a possible imperfect connection between the line on the posterior-inferior atrial wall and the right encircling; however, the completeness of the LA roof ablation provides a verifiable connecting scar between the 2 PVencircling ablations.

\section{Results}

From January 2005, 6 patients undergoing OPCAB (mean age, 70 years; range, 50-77 years) had the described ablation procedure. Atrial fibrillation (AF) lasting $43.3 \pm 27.7$ months (median, 47.0 months) was paroxysmal in 4 patients and permanent in 2 . The mean left atrial diameter was $45.6 \pm 7.9 \mathrm{~mm}$ (median, $43.5 \mathrm{~mm}$ ). No complications occurred. At $8.5 \pm 2.6$ months' follow-up (median, 9.8 months; range, $6-12$ months), 5 of 6 patients were in sinus rhythm, and 5 of 6 were off antiarrhythmic drugs. One patient with permanent AF had arrhythmia recurrence early after surgical intervention, but being lost to follow-up until data collection, he had not yet been given DC shock 6 months after the operation.

\section{Discussion}

The addition of the PLAC allows us to perform all left atrial ablations except the mitral line epicardially on the beating heart with a bipolar device. In addition to being applicable in patients with $\mathrm{AF}$ undergoing $\mathrm{OPCAB}$, this novel ablation technique has a potential role in lone AF treatment. For instance, such PLAC could be an adjunct to video-assisted bilateral PV isolation, as recently described by Wolf and colleagues. ${ }^{4}$

Of course, because this is a small series with relatively short follow-up, more patients and a longer observation period will be needed to validate the efficacy of this approach.

Unipolar devices allow drawing linear lesions more easily, especially in a close chest condition, but data supporting their epicardial efficacy are not nearly as solid as those on bipolar devices. ${ }^{5}$ Bipolar devices yield transmural ablations ${ }^{1-3}$ but have never been used clinically to connect the PV-encircling ablations on the beating heart because they are less ductile as a result of their design. ${ }^{2,3}$ 


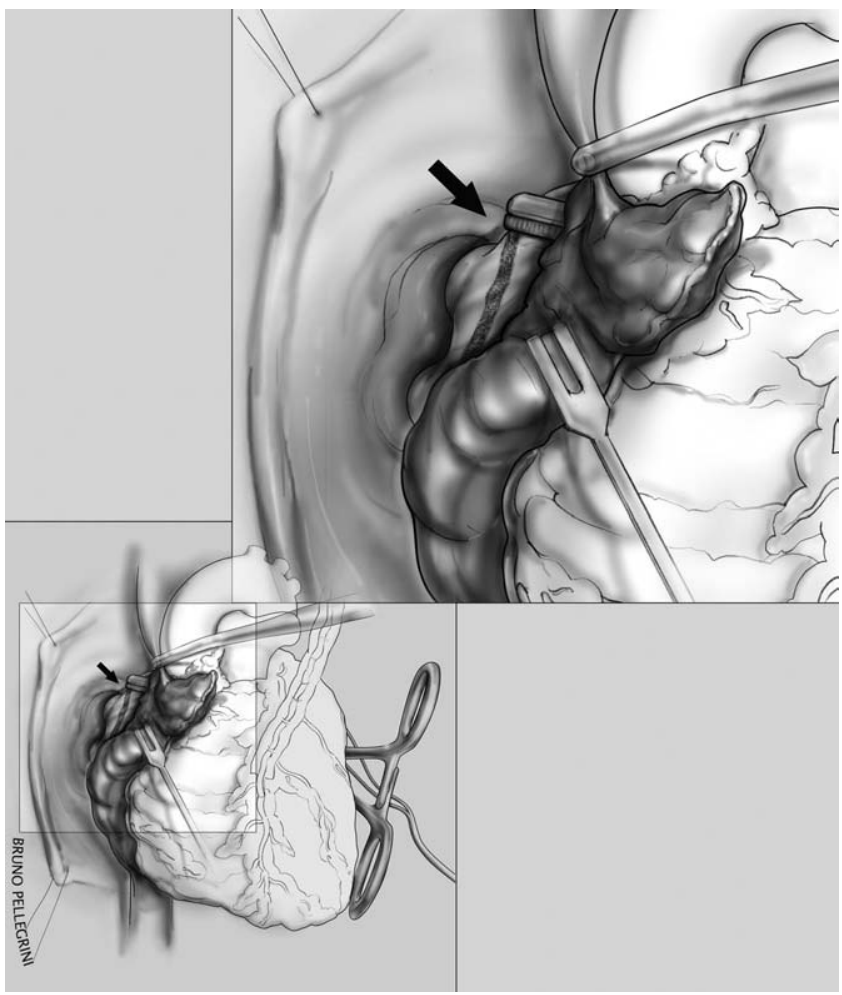

Figure 1. Anterior view of the heart: final position of the bipolar device. The detail in the right upper corner shows the tip of the upper jaw of the clamp clearly intersecting the right encircling ablation.

By expanding the spectrum of ablations feasible with bipolar devices epicardially, PLAC is meant to increase the efficacy of the existing beating-heart ablation techniques.

Starting from the described platform, the addition of a mitral line would enable a complete left atrial lesion set. Today, such a connection is only feasible with endocavitary approaches. However, alternative beating-heart epicardial options are under investigation.

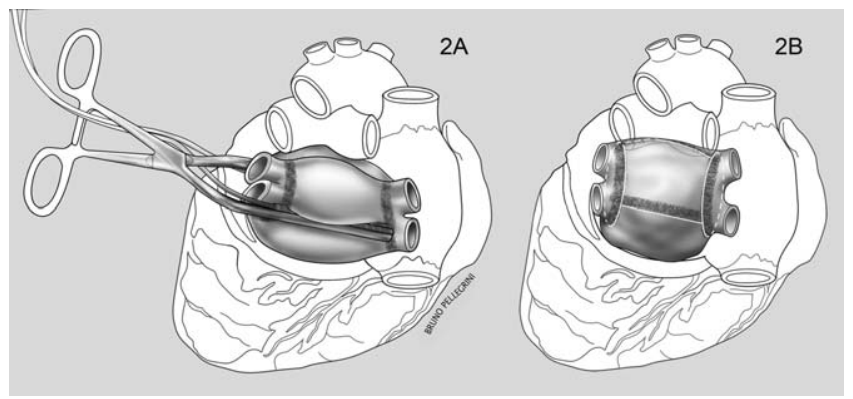

Figure 2. Posterior view of the heart (left atrium in gray): A, position of the bipolar device and its relation with the pulmonary veins during clamping; $B$, final set of ablations.

We thank Prof Bruno Pellegrini for his professionalism and patience in creating the artwork and Dr Francesco Maisano for his invaluable review of the manuscript.

\section{References}

1. Prasad SM, Maniar HS, Diodato MD, Schuessler RB, Damiano RJ Jr. Physiological consequences of bipolar radiofrequency energy on the atria and pulmonary veins: a chronic animal study. Ann Thorac Surg. 2003; 76:836-41

2. Benussi S, Nascimbene S, Calori G, Denti P, Ziskind Z, Kassem S, et al. Surgical ablation of atrial fibrillation with a novel bipolar radiofrequency device. J Thorac Cardiovasc Surg. 2005;130:491-7.

3. Gillinov AM, McCarthy PM, Blackstone EH, Rajeswaran J, Pettersson G, Sabik JF, et al. Surgical ablation of atrial fibrillation with bipolar radiofrequency as the primary modality. $J$ Thorac Cardiovasc Surg. 2005;129:1322-8.

4. Wolf RK, Shneeberger EW, Osterday R, Miller D, Merrill W, Flege JB $\mathrm{Jr}$, et al. Video-assisted bilateral pulmonary vein isolation and left atrial appendage exclusion for atrial fibrillation. J Thorac Cardiovasc Surg. 2005; 130:797-802.

5. Santiago T, Melo J, Gouveia RH, Neves J, Abecasis M, Adragao P, et al. Epicardial radiofrequency applications: in vitro and in vivo studies on human atrial myocardium. Eur J Cardiothorac Surg. 2003;24:481-6 